\title{
L'ADIASPIROMYCOSE (HAPLOMYCOSE)
}

\section{CHEZ LES MAMMIFERES DE LA SUISSE $\left(^{*}\right)$}

\author{
Par BERND HÖRNING et ERIKA HÖRNING-PEZENBURG
}

En 1942, Emmons et Ashburn décrivent une mycose pulmonaire chez diverses espèces de petits Rongeurs provenant des régions de steppes ou désertiques de l'Arizona (U.S.A.) et nomment le germe Haplosporangium parvum. Depuis, de telles mycoses pulmonaires (haplomycose) ont été retrouvées chez plusieurs espèces animales: Rongeurs, Insectivores et Mustélidés, provenant de pays répartis sur plusieurs continents (Dowding, 1947; Jellison, 1947, 1950, 1954 ; Mc Diarmid et Austwick, 1954 ; Menges et coll., 1954-55; Tevis, 1956; Bakerspigel, 1956-57 ; Jellison et coll., 1956, 1958, etc..., voir tableau I).

En suite de la diversité des espèces d'hôtes et de l'éloignement des différents lieux de capture, on a vu qu'il était erroné de croire que Haplosporangium parvum était seulement un parasite de régions désertiques bien délimitées.

La position systématique du germe parmi les champignons et la structure histologique des kystes pulmonaires, la grosseur des kystes, ont conduit à la certitude qu'il s'agissait en réalité de deux mycoses appartenant à des groupes différents (Dowding, 1947 ; Carmichaël, 1951 ; Breslau, 1955 ; Blank, 1957). Ciferri et Montemartini ont créé, en 1959, le genre monotype Emmonsia pour Haplosporangium parvum. Emmons et Jellison décrivent alors, en 1960, la nouvelle espèce : Emmonsia crescens, qui se différencie morphologiquement et par cultures de Emmonsia parva (syn. Haplosporangium parvum).

Ils proposent la désignation de adiaspiromycose pour les maladies pulmonaires des Mammifères dues aux parasites du genre Emmonsia.

Il est étonnant que des kystes mycosiques pulmonaires, atteignant jusqu'à $0,480 \mathrm{~mm}$., donc déjà visibles à l'œil nu, soient passés inaperçus des pathologistes et des mycologues jusqu'en 1942 [Jellison, Vinson et Borg, en 1961, trou-

(*) Ce travail a été effectué avec l'aide financière du « Fonds national suisse pour la recherche scientifique $»$ et de la \& Deutsche Forschungsgemeinschaft . Que ces deux institutions en soient ici remerciées. Nous tenons à remercier également M. le $\mathrm{D}^{r}$ W. L. Jellison, Rocky Mountain Laboratory, Hamilton, Montana (U.S.A.), qui a bien voulu contrổler morphologiquement et par culture notre diagnose, de l'intérêt qu'il a montré à notre travail ; le $\mathrm{Pr} \mathrm{D}^{\mathrm{r}} \mathrm{R}$. Fankhauser, de Berne, qui a bien voulu capturer et nous adresser des animaux pour autopsie, ainsi que MM. André Meylan et Gilbert Matthey, de Lausanne, de l'aide qu'ils nous ont apportée à la détermination des Rongeurs et Insectivores. 


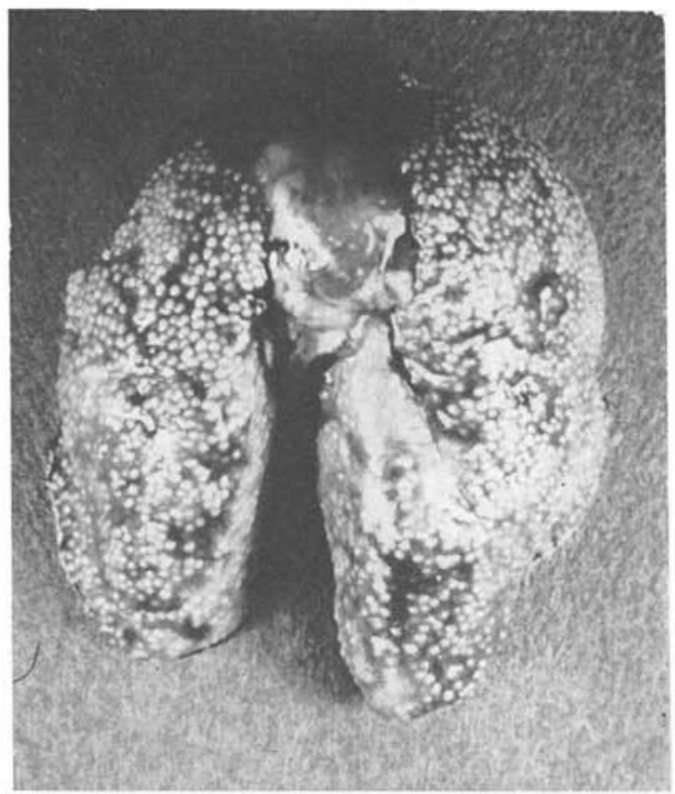

Fic. 1. - Poumons d'Arvicola scherman exilus avec très nombreux kystes d'Emmonsia crescens

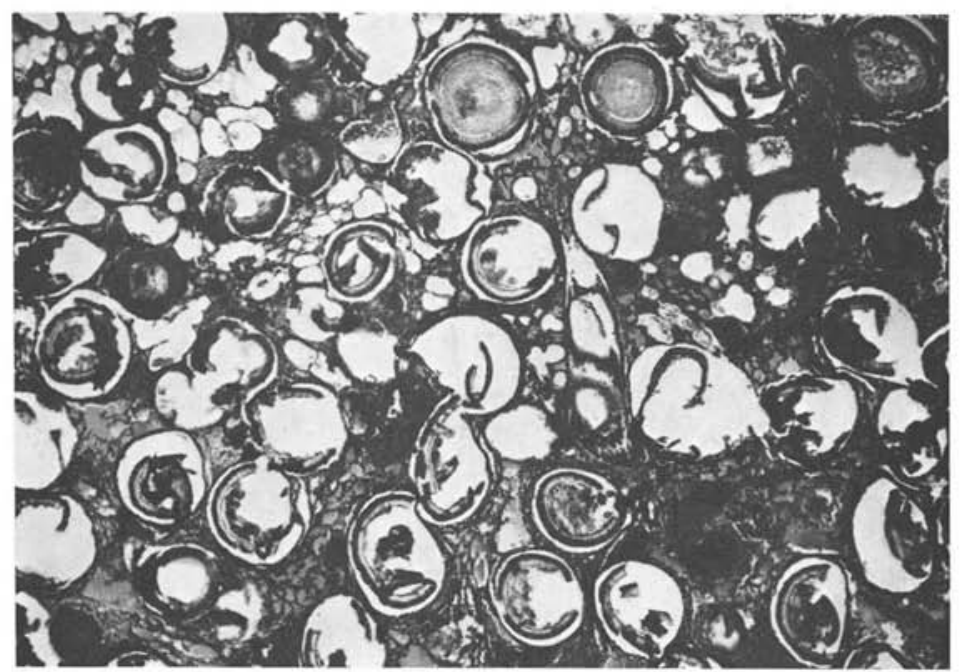

Fig. 2. - Même matériel (histologie) 

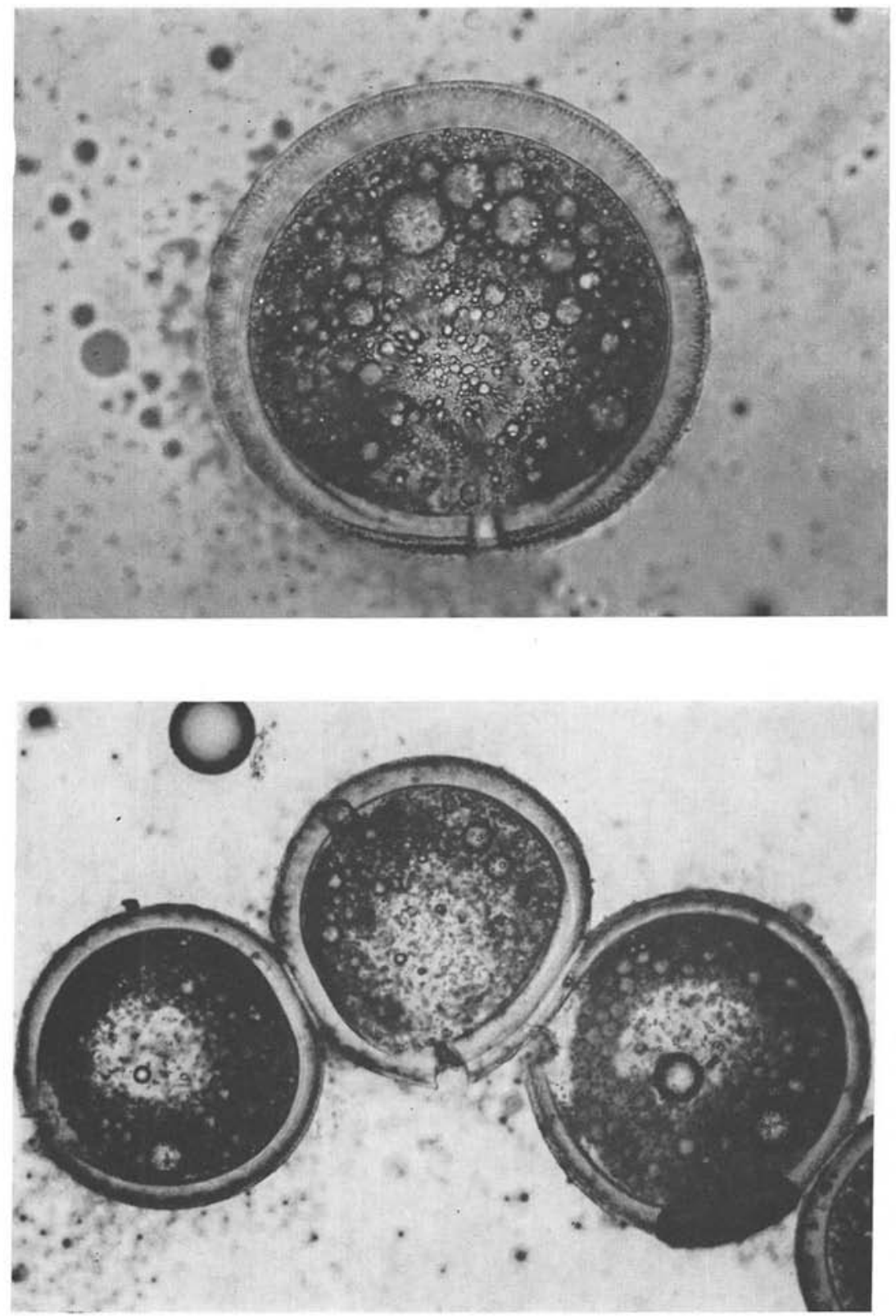

FIG. 3 et 4. - Emmonsia crescens (kystes). Poumon Arvicola scherman exitus (Photos Scholer, Bâle) 


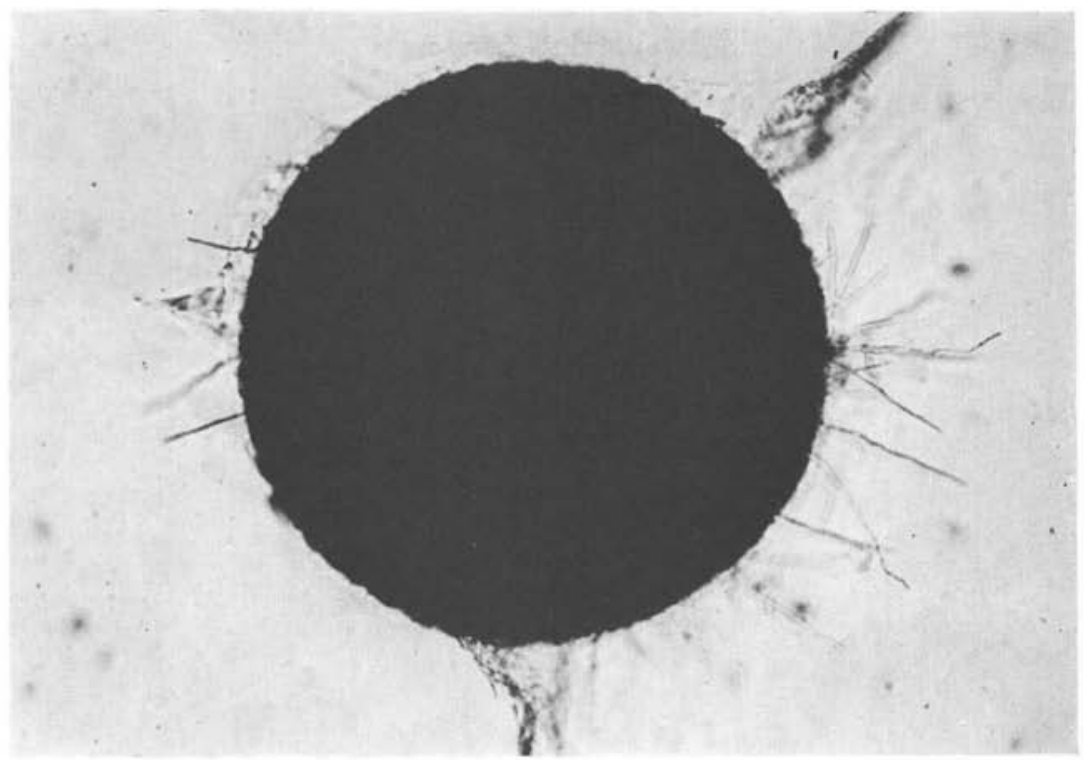

Fig. 5. - Emmonsia crescens. Culture sur lame de quatre jours (Photo Scholer, Bâle)

vent ces kystes mycosiques sur un poumon conservé de campagnol (Microtus agrestis), capturé en 1845 en Suède].

A l'instigation du $\mathrm{D}^{r} \mathrm{~W}$. L. Jellison, nous avons commencé, dès l'hiver 1960-61, les recherches de kystes d'Emmonsia chez les petits Mammifères terricoles. Ces recherches nous amenèrent, déjà à fin janvier 1961, à trouver cette affection chez six taupes (Talpa europaea), chez 54 animaux de cette espèce provenant d'Avenches (Vaud).

Les cas d'haplomycose que nous avons rencontrás depuis cette date en Suisse ou dans ses environs directs (Divonne, département de l'Ain, France) sont reportés dans le tableau II.

La maladie a été rencontrée partout où nous avons pu étudier des populations de petits Mammifères.

Nous avons trouvé des infestations chez la taupe (Talpa europaea), le mulot (Apodemus sylvaticus et $A$. flavicollis, car une détermination exacte n'est pas toujours possible), la souris domestique (Mus musculus), le campagnol Scherman (Arvicola scherman exitus qui, d'après Baumann, 1949, est la seule sousespèce existant au Nord des Alpes), le campagnol des champs et le campagnol agreste (Microtus arvalis et $M$. agrestis), le campagnol roussâtre (Evotomys glareolus), ainsi que chez la martre (Martes martes), ces animaux provenant des cantons de Berne, Genève, Vaud, Valais, ainsi que de Divonne (France). 
TABleau I. - Haplomycose : cas signalés jusqu'ici dans la littérature

\begin{tabular}{lll}
\hline Auteur & Provenance & Hôte \\
\hline
\end{tabular}

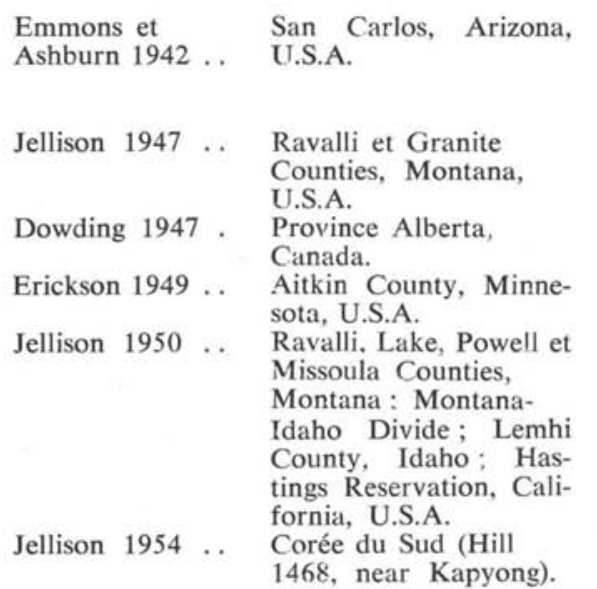

Perognathus baileyi, $P$. penicillatus, $P$. intermedius, Dipodomys merriami, Citellus harrisi, Onychomys torridus, Peromyscus eremicus.

Ochotona princeps.

Peromyscus maniculatus borealis, Sciurus hudsonicus baileyi.

Castor canadensis.

Sylvilagus nuttallii, Neotoma fuscipes, N. cinerea, Ochotona princeps, Peromyscus maniculatus, Ondatra zibethica, Tamiasciurus hudsonicus, Castor canadensis, Martes americana, Mephitis hudsonica, Mustela frenata, $M$. vison, $M$. erminea.

Apodemus agrarius, A. peninsulae, Clethrionomys rufocanus, Cricetulus triton, Rattus sp., « Musaraigne 》.

Mc Diarmid et Austwick 1954 .

Berkshire Downs;

Exeter, Devonshire, Talpa europaea.

Menges et $\mathrm{Ha}$ Angleterre.

bermann 1954;

Eastern Kansas, U.S.A.

Menges,

Habermann ei

Stains 1955 ....

Jellison 1956 ..

Gullgruva/Gävleborg, Suède.

Tevis $1956 \ldots$ Blandford, Dorset, Angleterre.

Bakerspigel 1956 Saskatoon,

Bakerspigel 1957 Saskatchewan, Canada. Ibidem

Jellison, Bell et coll. $1958 \ldots$.... Jellison 1958 .. Klamath Basin, Oregon, U.S.A.

Aomori, Kamikita Gun, Towada ; Miyagi, Ojika Gun, Kurumihana ;

Ehima, Nii Gun, Sumimo; Iwate, Iwate Gun, Matsuo, Japon ;

Mt. Meru, Tanganyika ;

Procyon lotor.

Nairobi, Kenya.

Apodemus flavicollis.

Apodemus flavicollis, A. sylvaticus, Clethrionomys glareolus, Microtus agrestis, Mus musculus, Talpa europaea, Sorex araneus, S. minutus, Erithacus rubecula $(* *)$.

Peromyscus maniculatus borealis.

Microtus pennsylvanicus drummondi. Microtus montanus.

Eothenomys smithii smithii, Apodemus speciosus speciosus.

Cricetomys sp., Ictonyx sp.

(**) Il semble que ce soit par erreur que Tevis cite Erithacus rubecula comme hôte, puisque son travail ne traite que des Mammifères. Il n'existe, à notre connaissance, aucun cas d'Oiseau hôte de kystes d'Emmonsia crescens. 
Tableau I. - Haplomycose : cas signalés jusqu'ici dans la littérature (suite)

\begin{tabular}{lll}
\hline Auteur & Provenance & Hôte \\
\hline
\end{tabular}

Jellison, Vinson et Helminen 1959 ; Jellison, Helminen et Vinson $1960 \ldots$ Jellison, Vinson et Holager 1960 Pezenburg 1960. Pezenburg (non publ., 1960) ... Jellison, Vinson et Borg 1961 ..

Jellison, Drouhet, Segretain. L'Héritier et Petter $1961 \ldots$.
Siilinjärvi ; Koijärvi ; Ile Korpholm ; Helsinki, Finlande.

Hamar, Norvège.

Berlin, Allemagne. Ibidem

Stockholm ; Lund ; Engelsberg/Västmanland ; Rättvik/Kopparberg,

\section{Suède.}

Argenton-sur-Creuse (Indre): St-Michel-enl'Herm (Vendée) : Brunoy (Seine-et-Oise); Axles-Thermes (Ariège), France.
Ondatra zibethica, Arvicola terrestris, Microtus agrestis.

Microtus sp., Arvicola terrestris.

Lepus europaeus.

Ondatra zibethica, Arvicola terrestris.

Apodemus sp., Microtus agrestis, Musaraigne (Neomys fodiens?), Lutra lutra.

Talpa europaea, Crocidura russula, Microtus arvalis, Clethrionomys glareolus.

Les kystes mycosiques n'ont pas été trouvés exclusivement dans les poumons, mais quelquefois aussi dans les ganglions lymphatiques pulmonaires, une fois dans le foie et une fois dans le diaphragme. Le nombre des kystes varie entre 1 et 250 environ.

Les poumons d'un Arvicola de Vouvry (Valais) (fig. 1 et 2) et d'une martre de Rovray (Vaud) contenaient une quantité presque innombrable de très gros kystes, bien que, à l'autopsie, les animaux puissent être considérés comme sains. Le $\mathrm{D}^{r}$ Jellison, sur le matériel provenant de cet Arvicola, a mesuré des kystes allant jusqu'à $0,580 \mathrm{~mm}$.

En conclusion, on peut dire que l'adiaspiromycose (haplomycose), due au champignon Emmonsia crescens Emmons et Jellison 1960, est relativement fréquente chez les petits Mammifères de la Suisse sud-occidentale et que des recherches étendues permettraient de la retrouver dans d'autres parties du pays. 
TABLEAU II. - Haplomycose : cas rencontrés en Suisse et dans la zone limitrophe immédiate en 1961-62

\begin{tabular}{|c|c|c|c|c|}
\hline $\begin{array}{l}\text { Localité } \\
\text { (canton) }\end{array}$ & Hôte & $\begin{array}{c}\text { Nombre } \\
\text { d'animaux } \\
\text { examinés }\end{array}$ & $\begin{array}{c}\text { Dont } \\
\text { positifs }\end{array}$ & Localisation \\
\hline \multirow{4}{*}{$\begin{array}{l}\text { Rosshäusern } \\
\text { (Berne) } \quad \ldots \ldots \ldots\end{array}$} & Talpa europaea & 21 & 1 & poumon \\
\hline & Apodemus sylvaticus & 20 & - & \\
\hline & Arvicola scherman exitus & 15 & 3 & poumon \\
\hline & Mus musculus & 2 & - & \\
\hline Drny (Vaud) ... & Microtus arvalis & 22 & 1 & poumon \\
\hline \multirow[t]{3}{*}{ Avenches (Vaud) } & Talpa europaea & 54 & 6 & poumon \\
\hline & Crocidura russula & 1 & - & \\
\hline & Epimys norvegicus & 1 & - & \\
\hline Rovray (Vaud) . & Martes martes & 1 & 1 & poumon \\
\hline Morges (Vaud) . & Microtus agrestis & 2 & 1 & poumon \\
\hline \multirow[t]{3}{*}{ Tannay (Vaud) . } & Arvicola scherman exitus & 3 & 1 & poumon \\
\hline & Microtus arvalis & 55 & 10 & poumon \\
\hline & Apodemus sylvaticus & 5 & - & \\
\hline \multirow{15}{*}{$\begin{array}{l}\text { Carouge } \\
\text { (Genève) } \ldots \ldots \\
\text { Genthod } \\
\text { (Genève) } \ldots \ldots \\
\text { Val d'Illiez } \\
\text { (Valais) } \ldots \ldots \ldots\end{array}$} & & & & \\
\hline & Microtus arvalis & 29 & 13 & poumon \\
\hline & Microtus arvalis & 15 & 4 & $\begin{array}{l}\text { poumon et } \\
\text { gangl. lymph. }\end{array}$ \\
\hline & & & & poumon et \\
\hline & Talpa europaea & 19 & 6 & gangl. lymph. \\
\hline & Sorex alpinus & 1 & - & \\
\hline & S. araneus & 13 & - & \\
\hline & S. minutus & 2 & - & \\
\hline & Neomys fodiens & 6 & - & \\
\hline & Rattus rattus & 4 & - & \\
\hline & Mus musculus & 1 & - & \\
\hline & Apodemus sylvaticus & 20 & 2 & poumon \\
\hline & Arvicola scherman exitus & 4 & 2 & poumon \\
\hline & Evotomys glareolus & 2 & - & \\
\hline & Microtus agrestis & 5 & - & \\
\hline \multirow{7}{*}{$\begin{array}{l}\text { Les Marécottes } \\
\text { (Valais), } \\
\text { Saint-Martin } \\
\text { (Valais) } \quad . . . . .\end{array}$} & Apodemus sylvaticus & 3 & 1 & poumon \\
\hline & Mus musculus & 1 & - & \\
\hline & Crocidura leucodon & & & \\
\hline & $\begin{array}{l}\text { Crocidura leucodon } \\
\text { Sorex araneus }\end{array}$ & $\begin{array}{l}8 \\
2\end{array}$ & - & \\
\hline & Evotomys glareolus & $\begin{array}{l}2 \\
5\end{array}$ & $\overline{1}$ & poumon \\
\hline & Apodemus sp. & 74 & 15 & poumon \\
\hline & Mus musculus & 39 & - & \\
\hline \multirow{6}{*}{ Vouvry (Valais). } & Talpa europaea & 3 & - & \\
\hline & Arvicola scherman exitus & 96 & 4 & poumon \\
\hline & Microtus arvalis & 12 & - & \\
\hline & Apodemus sylvaticus & 3 & - & \\
\hline & Mus musculus & 2 & - & \\
\hline & Talpa europaea & 7 & 2 & poumon et \\
\hline \multirow{8}{*}{$\begin{array}{l}\text { La Luette-sur- } \\
\text { Euseigne (Valais) }\end{array}$} & Sorex araneus & 11 & 一 & gangl. lymph. \\
\hline & Sorex minutus & 3 & - & \\
\hline & Sorex alpinus & 3 & - & \\
\hline & Crocidura russula & 1 & - & \\
\hline & Crocidura leucodon & 2 & - & \\
\hline & Mus musculus & 22 & 1 & poumon \\
\hline & Evotomys glareolus & 6 & 1 & poumon \\
\hline & Apodemus sp. & 105 & 6 & $\begin{array}{c}\text { poumon, ainsi } \\
\text { qu'un kyste } \\
\text { dans le foie } \\
\text { et le } \\
\text { diaphragme }\end{array}$ \\
\hline \multirow{2}{*}{$\begin{array}{l}\text { Divonne (Ain), } \\
\text { France ........ }\end{array}$} & Microtus arvalis & 3 & 2 & \\
\hline & Arvicola scherman & 1 & 1 & poumon \\
\hline
\end{tabular}




\section{RÉSUMÉ}

L'adiaspiromycose (haplomycose), due à Emmonsia crescens Emmons et Jellison 1960, a été trouvée dans les cantons suisses de Berne, Genève, Vaud, Valais, ainsi qu'à Divonne (Ain, France), chez des Insectivores: Talpa europaea, chez des Rongeurs : Arvicola scherman exitus, Microtus arvalis, M. agrestis, Evotomys glareolus, Apodemus sylvaticus, Mus musculus, et chez une martre: Martes martes.

\section{ZUSAMMENFASSUNG}

Adiaspiromycosis (Haplomycosis), verursacht durch Emmonsia crescens Emmons \& Jellison 1960, wurde bei Insectivoren (Talpa europaea), Nagetieren (Arvicola scherman exitus, Microtus arvalis, M. agrestis, Evotomys glareolus, Apodemus sylvaticus, Mus musculus) sowie bei einem Edelmarder (Martes martes) in den Schweizer Kantonen Bern, Genf, Waadt, Wallis, sowie in Divonne (Ain), Frankreich, gefunden.

\section{Bibliographie}

Ashburn (L. L.) et Emmons (C. W.), 1945. - Experimental Haplosporangium infection. Arch. of Pathology, 39, 3-8.

Bakerspigel (A.), 1956. - Haplosporangium in Saskatchewan Rodents. Mycologia, 48, 568-572.

- 1957. - Haplosporangium in an additional Rodent host, Microtus pennsylvanicus drummondi. Nature, London, 179, 875.

Baumann (F.), 1949. - Die freilebenden Säugetiere der Schweiz. Bern, 492 pp.

BLANK (F.), 1957. - Note on the chemical composition of the cell wall of Haplosporangium parvum. J. of Histochemistry and Cytochemistry, 5, 500-502.

Brestau (A. M.), 1955. - Comparative histochemical studies on Coccidioides immitis and Haplosporangium parvum. J. of Histochemistry and Cytochemistry, 3, 141-147.

Carmichä̈L (J. W.), 1951. - The pulmonary fungus, Haplosporangium parvum. II. Strain and generic relationships. Mycologia, 43, 605-624.

Ciferri (R.) et Montemartini (A.), 1959. - Taxonomy of Haplospotangium parvum. Mycopathologia Mycologia appl., 10, 303-316.

Dowding (E. S.), 1947a. - Haplosporangium in Canadian Rodents. Mycologia, $39,372-373$.

- $1947 \mathrm{~b}$. - The pulmonary fungus, Haplosporangium parvum and its relationship with some human pathogens. Canad. J. Research, 25, 195-206.

Emmons (C. W.), 1948. - Coccidioidomycosis and haplomycosis. Proc. $4^{\text {th }}$ Internat. Congr. Trop. Med, and Malaria, Washington, 2, 1278-1286. 
et Ashburs (L. L.), 1942. - The isolation of Haplosporangium parvum n. sp. and Coccidioides immitis from wild Rodents. Their relationship to coccidioidomycosis. Public Health Reports, 57, 1715-1727.

et Jellison (W. L.), 1960. - Emmonia crescens sp. n. and adiaspiromycosis (haplomycosis) in Mammals. Annals of the New York Acad. Sci., 89, 91-101.

Erickson (A. B.), 1949. - The fungus (Haplosporangium parvum) in the lungs of the beaver (Castor canadensis). J. of Wildlife Management, 13, 419-421.

JeLlison (W. L.), 1947. - An undetermined parasite in the lungs of a rock Rabbit, Ochotona princeps Richardson (Lagomorpha: Ochotonidae). Proc. Helminthol. Soc. Washington, 14, 75-77.

- 1950. - Haplomycosis in Montana Rabbits, Rodents and Carnivores. Public Health Reports, 65, 1057-1063.

1954. - The presence of a pulmonary fungus in Korean Rodents. Publit: Health Reports, 69, 996-997.

- 1956. - Haplomycosis in Sweden. Nordisk Veterinaermedicin, 8, 504-506.

- 1958. - Haplomycosis in Japan and Africa. Mycologia, 50, 580-583.

- Bell (J. F.), Vertrees (J. D.), Holmes (M. A.), Larson (C. L.) et Owen (C. R.), 1958. - Preliminary observations on diseases in the 1957-1958 outbreak of Microtus in western United States. Transact. 23rd North

American Wildlife Conference, 137-145.
Drouhet (E.), Segretain (G.), L'Héritier (M.) et Petter (F.), 1961. Adiaspiromycose (haplomycose) chez les Mammifères sauvages en France. Annales Institut Pasteur, Paris, 100, 747-752.

Helminen (M.) et Vinson (J. W.), 1960. - Presence of a pulmonary fungus in Rodents in Finland. Ann. Med. Exper. Fenn., 38, $\mathrm{n}^{\circ} 4,8 \mathrm{pp}$.

Vinson (J. W.) et Bong (K.), 1961. - Adiaspiromycosis (haplomycosis) in Sweden. Acta Vet. Scandinav., 2, 178-184.

et Helminen (M.), 1959. - Haplomykoosi, sientauti piisamissa. Suomen Riista, 13, 184-185.

- - et Holager (E.), 1960. - Haplomycosis in Norway. Acta Path. et Microbiol. Scandinav., 49, 480-484.

Mc Diansid (A.) et Austwick (P. K. C.), 1954. - Occurrence of Haplosporangium parvum in the lungs of the mole (Talpa europaea). Nature, London, 174, 843.

Menges (R. W.) et Habermann (R. T.), 1954. - Isolation of Haplosporangium parvum from soil and results of experimental isolation. Amer. J. Hyg., 60, 106-116.

- - et Stains (H. J.), 1955. - A distemper-like disease in raccoons and isolation of Histoplasma capsulatum and Haplosporangium parvum. Transact. Kansas Acad. Sci., 58, 58-67.

Pezenburg (E.), 1960. - Haplomykose beim Hasen. Zentralbl. Bakt., I. Abt., Orig., 178, 140 .

Tevis (L.), 1956. - Additional records of Haplosporangium parvum in Mammals in Britain. Nature, London, 177, 437.

(Institut Galli-Valerio, Lausanne, Suisse. Direct ${ }^{r}$ : $\mathrm{D}^{r}$ G. BouvIER) 